\title{
Implementation of an HIV-1 Triple-Target NAT Assay in the Routine Screening at Three German Red Cross Blood Centres
}

\author{
Silke De Zolt ${ }^{\mathrm{a}}$ Rolf Thermann ${ }^{\mathrm{a}}$ Thorsten Bangsow ${ }^{\mathrm{a}}$ Lutz Pichl $^{\mathrm{b}}$ Benjamin Müller ${ }^{\mathrm{b}}$ \\ Christine Jork $^{c}$ Marijke Weber-Schehl ${ }^{d}$ Doris Hedges ${ }^{d}$ Ingo Schupp ${ }^{a}$ Patrick Unverzagt $^{a}$ \\ Katrin de Rue ${ }^{\mathrm{a}}$ W. Kurt Roth ${ }^{\mathrm{a}}$ \\ a Gesellschaft zur Forschung, Entwicklung und Distribution von Diagnostika im Blutspendewesen mbH (GFE Blut), Frankfurt/M., Germany; \\ ${ }^{b}$ DRK Blutspendedienst West, Hagen, Germany; \\ ${ }^{\mathrm{c}}$ DRK Blutspendedienst NSTOB, Springe, Germany; \\ ${ }^{\mathrm{d}}$ Blutspendedienst des Bayerischen Roten Kreuzes, Wiesentheid, Germany
}

\section{Keywords}

Blood safety · HIV-1 variants · NAT · Multi-target PCR . HIV-1 triple-target NAT · High throughput testing

\section{Summary}

Background: Blood product safety was significantly improved by the introduction of NAT testing in the late 1990s, resulting in a strong decrease of transfusion-transmitted infections (TTIs). Due to the occurrence of HIV-1 NAT test failures as a consequence of mismatch mutations in the amplicon regions of mono-target NAT assays, the Paul Ehrlich Institute mandated the implementation of multi-target NAT assays for HIV-1 in 2014. Commercial suppliers mostly developed dual-target NAT assays, with only one implementing a triple-target NAT assay. Methods: The HIV-1 triple-target NAT assay v3 (GFE Blut) was tested on mutated specimens and synthetic DNA bearing mutations that resulted in sample underquantification or false-negative test results. In addition, data from 2 years routine testing at three German Red Cross Blood centres were analysed. Results: The HIV-1 triple-target PCR could compensate for all mutations tested and could compensate the loss of one amplicon without a significant loss of sensitivity. Data from 2 years routine testing showed a solid performance. Conclusion: The HIV-1 triple-target v3 assay (GFE Blut) can compensate mutations in target sequences better than a dual-target assay and is applicable to high-throughput screening, thus increasing blood product safety.

\section{Introduction}

Nucleic acid amplification technology (NAT) was first introduced in the mid-1990s for fractionation plasma followed by the testing of labile blood components at the end of the 1990s [1-3]. In the early days, NAT testing of blood donations was a manual inhouse process since neither commercial assays nor automated test platforms were available. Therefore, NAT testing of blood donations was restricted to only a few countries that had the capabilities to develop and implement those tests.

Blood centres in Germany started NAT screening for hepatitis $\mathrm{C}$ virus (HCV), hepatitis B virus (HBV) and human immunodeficiency virus type 1 (HIV-1) already in 1997 on a voluntary basis [2, 3]. Besides the mandated HCV NAT (1999) and HIV-1 NAT (2004), tests for human immunodeficiency virus type 2 (HIV-2), hepatitis A virus (HAV) and parvovirus B19 were introduced in 2000 on a voluntary basis.

First commercial NAT assays for HCV and HIV-1 became available in 1999, but it took many more years to have commercial assays for HBV, HIV-2, HAV and parvovirus B19 introduced as well. In 2005, the German Red Cross decided to transform their manual in-house approach into a fully automated NAT testing system by establishing a new commercial company, the 'Gesellschaft zur Forschung, Entwicklung und Distribution von Diagnostika im Blutspendewesen mbH' (GFE Blut). It took the GFE Blut 5 years to launch its first highly automated NAT testing system autoX for high-throughput blood donation testing. The development was guided by the experience of more than 9 years of in-house NAT testing at the German Red Cross blood centres, with millions of donations tested for the broad spectrum of human pathogens rele-

\section{KARGER}

Fax +497614520714

\section{() 2016 S. Karger GmbH, Freiburg}

$1660-3796 / 16 / 0433-0183 \$ 39.50 / 0$
Prof. Dr. W. Kurt Roth

Gesellschaft zur Forschung, Entwicklung und Distribution von Diagnostika im Blutspendewesen mbH (GFE Blut)

Altenhöferallee 3

60438 Frankfurt, Germany

kurt.roth@gfeblut.de 
vant for blood donation safety: HCV, HBV, HIV-1/2, HAV, and parvovirus B19 [2, 4]. One of the key features of the autoX system is the parallel processing and analysis of these 6 viruses within one run and, due to the multi-dye approach, without the need for successive discriminatory PCRs.

NAT testing proved to be very efficient in its intended use to minimise the pre-seroconversion window phase even when pools of 96 samples are processed, as commonly performed by the big German blood centres [5]. Germany has very low frequencies of virus-infected donors [6] which allows pooling with lower input and reduced sensitivity. The validity of this strategy is well supported by the rate of transmissions that were observed. For HCV, only 1 transmission by a blood donation in 80 million donations tested has been observed since the mandated introduction of HCV NAT testing in Germany in 1999 [7]. The transmission could probably not have been avoided by applying the more sensitive single donation testing, since the viral load was below the detection limit of even the most sensitive single donation tests available [7].

The same strategy has also paid off for HIV-1 donation screening. Only two HIV-1 transmissions have been observed since the mandated introduction of HIV-1 NAT in 2004, and both test failures (case 1 and case 3 , according to [8]) were due to mutations in the primer or probe binding regions of the respective HIV-1 isolates, leading to test failures despite high viral loads [8-10]. Mutations happened to be in different genomic regions of HIV-1, and both donations escaped antibody testing due to pre-seroconversion phase. In one of the cases, mutations occurred in the gag region of the HIV-1 genome [11], which prompted the manufacturer to move the target region to the HIV-1 long terminal repeat (LTR), which was expected to be more conserved. However, in the second case the viral isolate contained numerous mutations within the LTR region, thus resulting in a second test failure and subsequent transmission of the virus. As for $\mathrm{HCV}$, the transmissions could probably not have been avoided by single donation testing since in both cases mutations in the target regions resulted in a serious under-quantification (factor up to 100 , case 1 ) or a non-functional PCR (case 3), again supporting the validity of the pool testing approach in Germany [8].

At least additional 17 cases of HIV-1 test failures have been described [9], with no transmission because all donations showed a positive result in the serological tests and were discarded prior to transfusion. Re-testing of those samples revealed that HIV-1 NAT tests with two different target regions did not fail to detect these virus variants but showed partially a reduced sensitivity if one target region failed in amplification [8, 9].

These observations resulted in a decision by the Paul Ehrlich Institute (PEI) to mandate that all blood components must be tested by HIV-1 NAT systems that are able to exclude or compensate for the potential underestimation or non-recognition of the respective target region. A potential approach could be a NAT test with two or more different target regions. For each individual target region the limit of detection $\left(\mathrm{LOD}_{95 \%}\right)$ must fulfil the minimal requirement of 10,000 IU/ml per individual donation [12]. The federally mandated measures had to be implemented by January 1, 2015 .
GFE Blut decided to develop a triple-target assay to gain more assay robustness and safety, since it has been shown that even dualtarget NAT assays had a reduced sensitivity if the amplification of one target region fails due to mutations in the respective target region. This triple-target HIV-1 PCR assay (HIV-1 v3 PCR) is, as a part of the Virus Screening PCR Kit version 1.3 (VSPK v1.3), in routine use since 2014 .

Alongside with key performance characteristics of the tripletarget HIV-1 PCR assay, we will discuss the analysis of routine screening data obtained from three big German blood centres who have implemented the assay in their routine donor NAT testing with more than 3.5 million samples tested with the HIV-1 v3 PCR.

\section{Material and Methods}

Test Sites and autoX System

The data sets for the HIV-1 triple-target PCR were collected between January 2014 and December 2015 at three different test sites: i) DRK Blutspendedienst NSTOB, Springe, Germany, ii) DRK-Blutspendedienst West, Hagen, Germany and iii) Blutspendedienst des Bayerischen Roten Kreuzes, Wiesentheid, Germany.

The HIV-1 NAT screening was performed using the autoX 1.5 or 2.0 platform (GFE Blut, Frankfurt/M., Germany) with the Virus Screening PCR Kit version 1.3 (GFE Blut) in a minipool (MP) format of up to 96 samples per pool. Test sites A and B are using the autoX 2.0 platform with the LightCycler $480^{\circledR}$ Realtime PCR system (Roche Diagnostics Ltd., Rotkreuz, Switzerland); test site C is using the autoX 1.5 platform with the Applied Biosystems ${ }^{\text {TM }} 7500$ Real-Time PCR System (Applied Biosystems, Foster City, CA, USA). For blood donation screening, MPs of up to $96 \mathrm{~K} 2$ EDTA plasma samples were constituted by pooling $0.1 \mathrm{ml}$ of plasma aliquots from individual donations. After addition of the internal control (IC Kit; GFE Blut), the MPs were centrifuged for $1 \mathrm{~h}$ at $58,510 \times g$ in a centrifuge (Avanti, Beckman, Munich, Germany) with a fixed-angle rotor (Beckman) to enrich viral particles. Subsequently, supernatant was decanted. The tubes containing the pellets were loaded onto the autoX platform, and nucleic acids were automatically extracted with the autoX Extraction Kit (GFE Blut), followed by realtime PCR setup, amplification and detection using the Virus Screening PCR Kit (VSPK v1.3; GFE Blut) according to the manufacturer's instructions.

\section{Determination of the $L O D_{95 \%}$ and Statistical Analysis}

To determine the $\mathrm{LOD}_{95 \%}$ for a pool of 96 samples, serial dilutions of the WHO International Standard for HIV-1 (10/152) were prepared in pooled, negative human K2 EDTA plasma with 7 different dilution levels and 29 or 30 replicates each.

To determine the $\mathrm{LOD}_{95 \%}$ for the triple-target PCR with the loss of one amplicon region in comparison to a dual-target PCR, serial dilutions of a HIV-1 cell culture supernatant calibrated against the WHO International Standard for HIV-1 (97/650) were prepared in pooled, negative human K2 EDTA plasma with 7 different dilution levels and 36 or 42 replicates each. The loss of one amplicon region in the PCR was simulated by exclusion of the corresponding probe in the reaction mixture.

The data were used to calculate the $\mathrm{LOD}_{95 \%}$ by PROBIT analysis (IBM SPSS Statistics 19; IBM Corp, Armonk, NY, USA).

\section{Phylogenetic Analysis}

Phylogenetic analysis was done using maximum likelihood statistics with MEGA6 [13] using default settings. As a basis for phylogenetic analysis, a prebuild HIV-1 reference alignment (HIV1_REF_2010_genome_DNA) from the HIV-1 database (Los Alamos, NM; www.hiv.lanl.gov) was downloaded and the two sequences of case 4 and 5 isolates (according to [9]) as well the sequence of the WHO international standard (KJ019215) were manually integrated. The alignment was further manually edited using Jalview 2 (www.jalview.org [14]). 
To show robustness of the phylogenetic analysis, the trees were calculated using bootstrapping with 100 replicates, and the analysis was repeatedly done with different parts of the alignment (e.g. sole the gag, pol, env ORF regions or together with a part of the LTR region) using different statistical methods (UPGMA, neighbour joining, maximum likelihood). Insertions and variable regions were removed manually from the alignments before phylogenetic analysis was performed.

\section{Subtype Detection}

Serial dilutions using synthetic DNA sequences $\left(\right.$ gBlocks $^{\circledR}$; Integrated DNA Technologies, Coralville, IA, USA), which were designed to represent relevant subtypes of HIV-1 and published sequences with rare mutations including case 4 isolate (according to [9]), were amplified by the single PCRs (LTR, gag, pol). For each of the 7 concentrations of gBlocks copies per reaction (cop/rxn) 4 replicates were tested and PROBIT analysis was done (IBM SPSS Statistics 19).

To analyse Ct values, 1.000 cop/rxn of gBlocks were used in the single PCRs (LTR, $g a g, p o l$ ). For every subtype or sequences with rare mutations the Ct values for the single PCRs were compared to each other.

\section{Results}

Analysis of Known HIV-1 Test Failures and Implications on the HIV-1 Triple-Target Assay Design

The PEI investigated 6 cases of HIV-1 NAT test failures in more detail, including sequence analysis of the primer and probe binding sites [9]. The samples were further analysed by approved qualitative and quantitative HIV-1 NAT assays for blood donation screening and viral load testing. The data showed that all test failures occurred due to mutations in the primer and probe binding sites, although the isolates were all B subtypes which should be fully covered by all NAT tests under investigation. Since highly conserved regions are rare in the HIV-1 genome, the assay design of almost all NAT assays focuses on short sequences in the LTR, gag and pol/integrase regions. Viral isolates from cases 1 and 2 (according to [9]) were mutated in the gag region leading to failures of gag-based assays [8]. The manufacturer of the gag-based assay that failed on those viral isolates re-designed the assay to target the LTR region, but then new isolates with highly mutated LTR sequences were identified (cases 3-6), leading again to test failures [9]. The pol region seems to be more conserved, since one test with a pol-based amplicon readily amplified all mutant isolates. But most importantly, dual-target assays, one targeting the gag/ LTR regions and one targeting the LTR/pol regions, detected all isolates with an only moderately reduced sensitivity [9].

Three of the described 6 test failures (cases 4-6, according to [9]) happened to the LTR-based HIV-1 blood screening NAT assay of GFE Blut. Fortunately, the release of the contaminated blood products could be prevented. In October 2010, 2 blood donors (case 4 and 5, according to [9]) tested NAT-negative, antibody-positive at two different test sites in Germany which employed the VSPK v.1.1 (GFE Blut), despite a high viral load of 200,000 and 2,000 IU/ml. We sequenced almost the entire genome of both isolates and found a set of four mutations in the sense primer binding site which were identical in both samples and finally caused the failure of the assay. Sequence analysis of the two isolates with jpHMM (http://jphmm.gobics.de [15]) for genotyping and recombinant detection confirmed subtype $\mathrm{B}$ and gave no hint to a recombination event. Furthermore, BLAST analysis (http:// blast.ncbi.nlm.nih.gov/ [16]) showed only a moderate similarity of about $92 \%$ with other subtype B isolates. Phylogenetic analysis of the sequences revealed that these isolates were genetically related subtype B isolates but grouped on a separate branch in the subtype B clade (fig. 1). The sequences of the two isolates were submitted to the GenBank sequence database with the following accession numbers: KU612900 (isolate 4) and KU612901 (isolate 5).

The VSPK v.1.1 was replaced immediately by a new version (VSPK v.1.2) with a re-designed primer set to the LTR region, taking into account these previously unknown mutations. However, in 2012 a new isolate came up in a donation from a female first-time donor (case 6, according to [9]) that was NAT-negative in the new VSPK assay but antibody-positive. Upon sequencing of the LTR region, a deletion of $56 \mathrm{nt}$ in the so far most conserved probe binding site could be identified affecting almost all LTR-based NAT assays $[9,10]$. Moreover, the PEI performed further sequencing analysis revealing an additional $32 \mathrm{nt}$ insertion upstream of the deletion [9]. These findings, together with the data published by the PEI, prompted us to develop a triple-target NAT assay instead of a dualtarget NAT as suggested by the PEI as a minimum requirement [12].

In-depth sequence analysis and in-silico PCR design enabled us to choose the optimal primer and probe target sequences within the LTR, pol and gag regions, which were the first choice due to conservation and primary sequence composition. Using synthetic DNA sequences (gBlocks), which were designed to represent relevant subtypes of HIV-1 and published sequences with rare mutations, including one of the new isolates (case 4, according to [9]), it became evident that there were substantial differences in the $\mathrm{LOD}_{95 \%}$ and $\mathrm{Ct}$ values among the different sequences represented by the gBlocks for every single PCR (fig. 2). The results reflect the mutation rate of the target region as expected. No amplification was detected for the gag amplicon with the group $\mathrm{N}, \mathrm{O}$ and $\mathrm{P}$ subtypes which is not surprising since the gag region is the worst conserved among the three (black bars, fig. 2). However, the other tested sequence variants produced robust analytical sensitivities (between 1.0 and 3.1 cop/rxn). Although mutations in primer regions show the expected shift to higher Ct values (fig. 2), this does obviously not impair sensitivity, pointing to a good mismatch tolerance of the assay.

The failure of the gag amplicon to detect the genotypes $\mathrm{O}, \mathrm{N}$ and $\mathrm{P}$ is a problem most probably shared by all multi-target HIV-1 NAT assays, thus reducing some assays just being a mono-target assay on the affected HIV-1 genotypes. In contrast, our triple-target approach still maintains a dual-target approach for these rare genotypes of HIV-1 as well as for the previously described isolate with the 56-nucleotide deletion which cannot be detected by the re-designed LTR-PCR included in the triple-target assay (case 6, data not shown). In addition to the gBlocks approach, we also tested the triple-target PCR on available genotype panels at a $3 \times$ $\mathrm{LOD}_{95 \%}$ without any test failure (data not shown).

The results obtained from the gBlocks experiments and the tested genotype panels clearly show the potential of the triple-target approach, namely the high level of safety in the detection of mutated virus isolates or on rare genotypes. 
Fig. 1. A Phylogenetic (maximum likelihood) tree of an alignment of the gap-pol-env genomic region (nt 790-8795 on reference clone HXB2 (K03455)) of the case 4 and 5 isolate sequences and HIV-1 reference sequences. B Subtree of (A) of the subtype B clade with bootstrap values. Phylogenetic analysis was done using maximum likelihood (ML) statistics with MEGA6 [13] using default settings. As a basis for phylogenetic analysis a prebuild HIV-1 reference alignment (HIV1_REF_2010_genome_DNA) from the HIV-1 database (Los Alamos, NM; www.hiv. lanl.gov) was downloaded and the two sequences of case 4 and 5 isolates, as well the sequence of the WHO international standard (KJ019215) were manually integrated and the alignment further manually edited using Jalview 2 (www.jalview. org [14]). To show robustness of the phylogenetic analysis, the trees were calculated using bootstrapping with 100 replicates and the analysis was repeatedly done with different parts of the alignment (e.g. the gag, pol, env ORF regions alone or with a part of the LTR region) using different statistical methods (UPGMA, neighbour joining, maximum likelihood). Insertions and variable regions were removed manually from the alignments before phylogenetic analysis was performed.
A

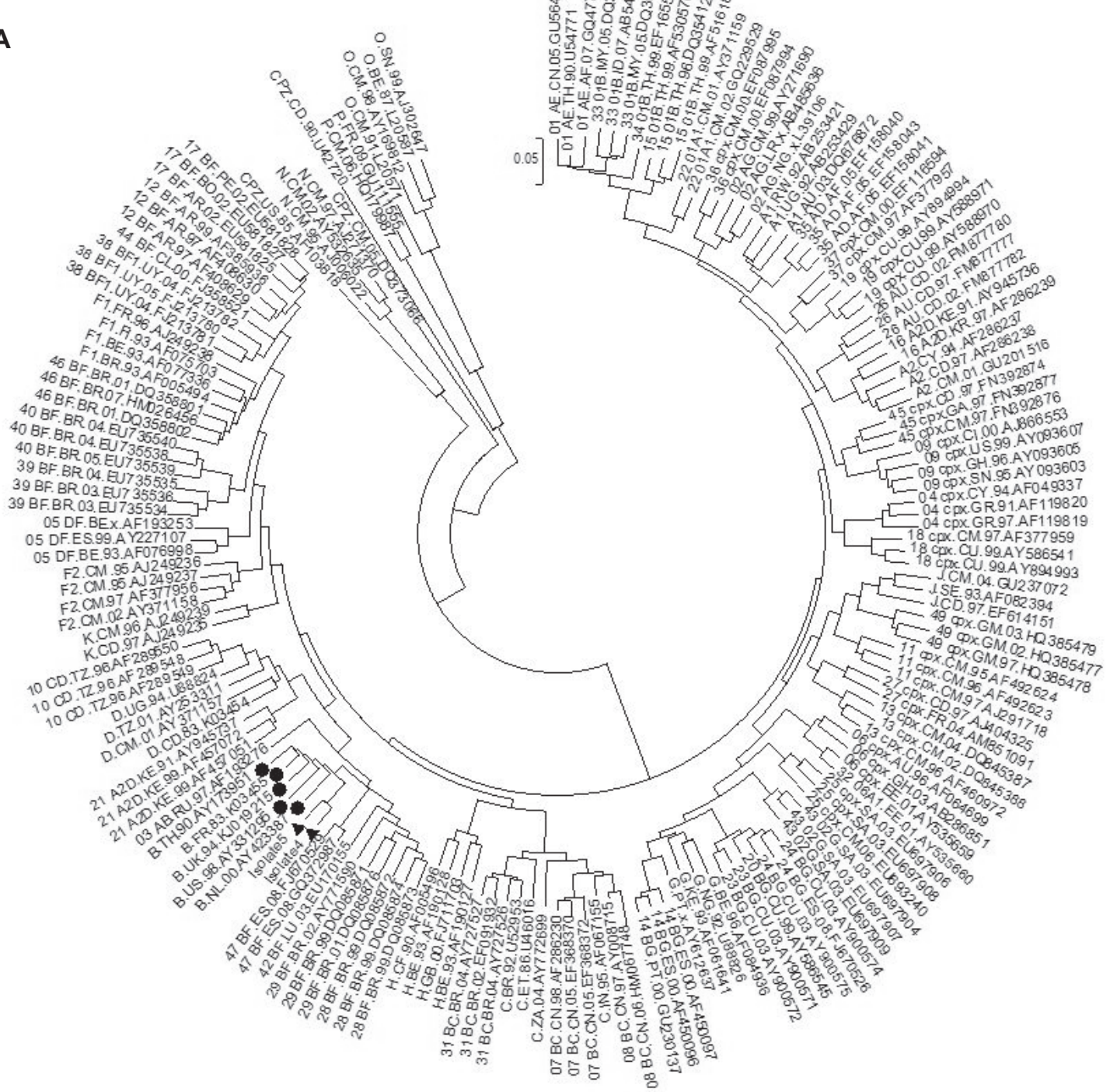

B

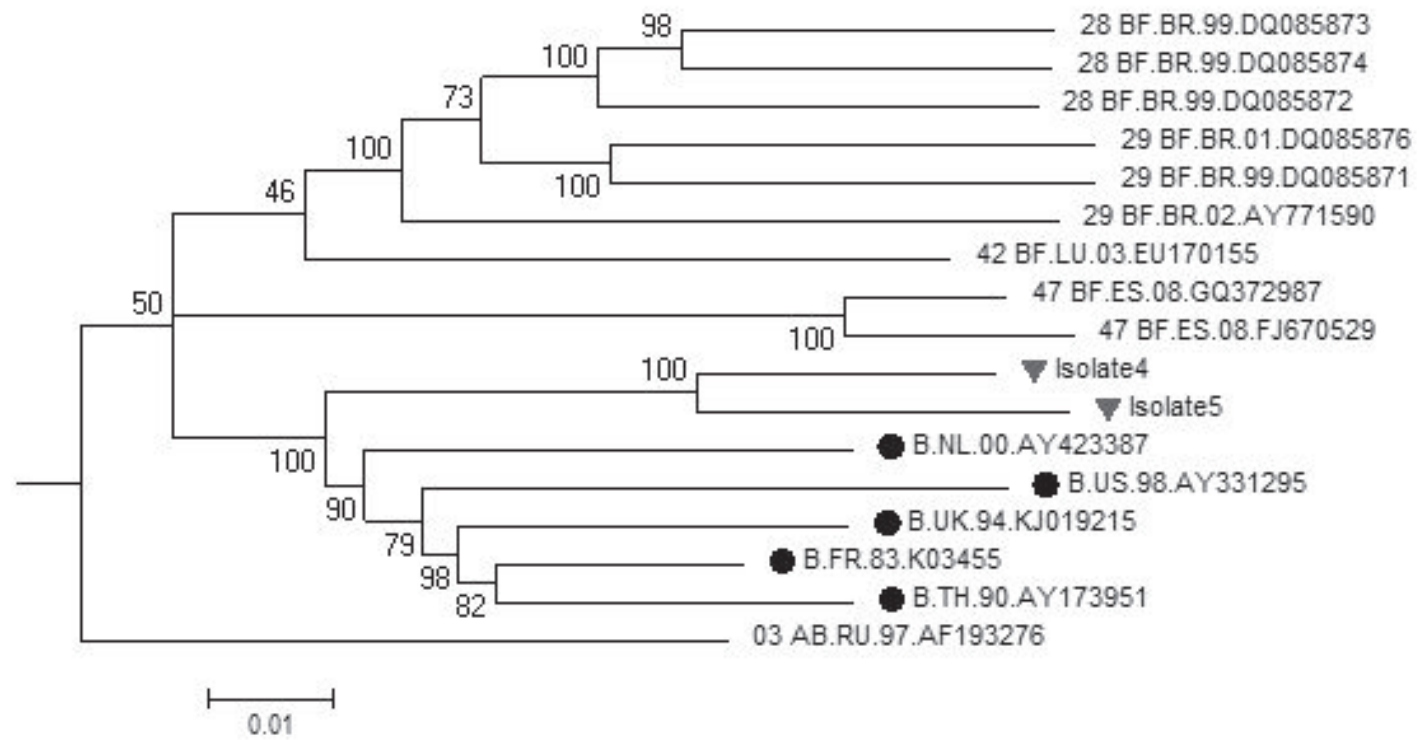




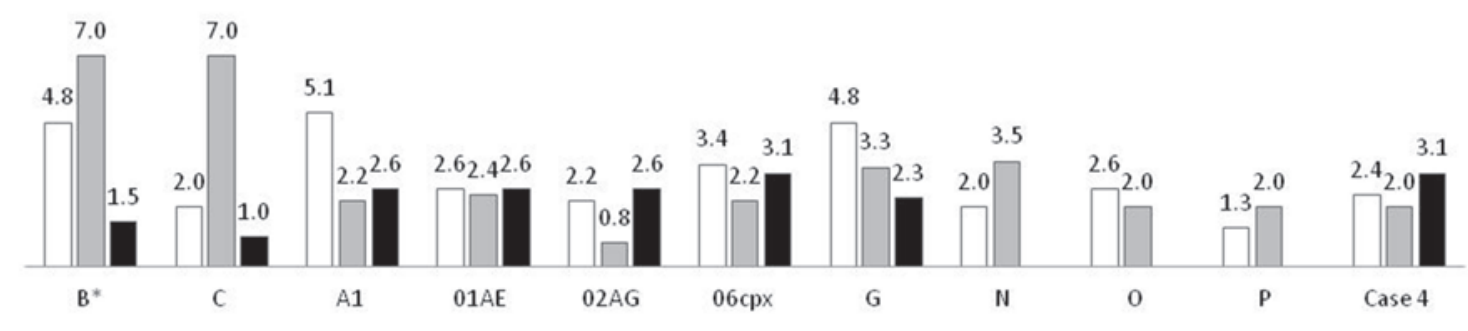

B

Fig. 2. A $95 \%$ limit of detection for the single amplicons. The described subtypes are represented by gBlocks. B Ct values for the single amplicons obtained from gBlocks for a concentration of $1,000 \mathrm{cop} / \mathrm{rxn}$.

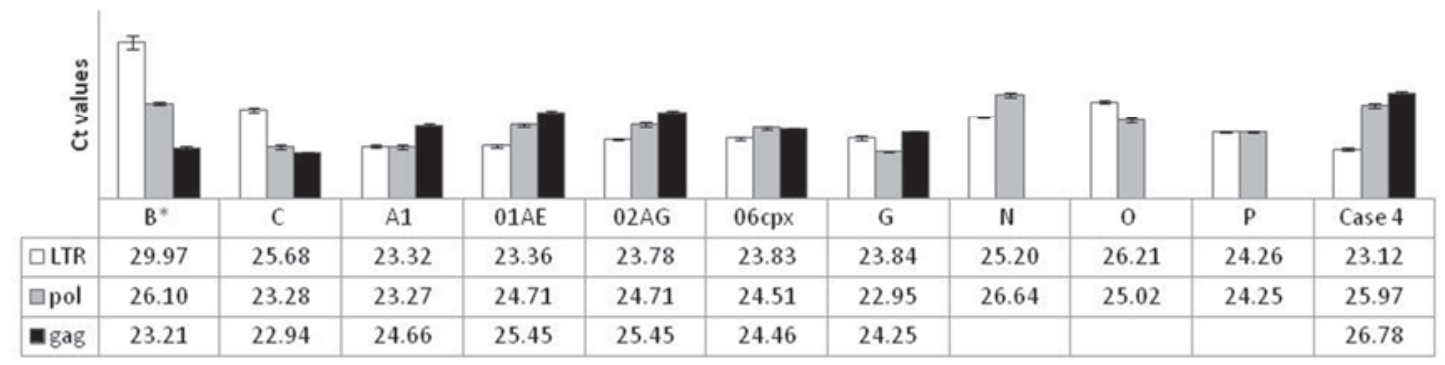

*no consensus sequence, published highly mutated sequence of subtype B

Table 1. Comparison of the $\mathrm{LOD}_{95 \%}$ and confidence interval (CI) of a dual- to a triple-target PCR for the simulated loss of one amplicon region

\begin{tabular}{|c|c|c|c|c|c|c|c|c|}
\hline & \multicolumn{2}{|l|}{ A } & \multicolumn{2}{|l|}{ B } & \multicolumn{2}{|l|}{$\mathrm{C}$} & \multicolumn{2}{|l|}{$\mathrm{D}$} \\
\hline & triple & dual & triple w/o pol & dual w/o pol & triple w/o LTR & dual w/o LTR & triple w/o gag & dual \\
\hline $\mathrm{LOD}_{95 \%}, \mathrm{IU} / \mathrm{ml}$ & 279 & 371 & 398 & 618 & 319 & 728 & 329 & 371 \\
\hline $\mathrm{CI}, \mathrm{IU} / \mathrm{ml}$ & $207-608$ & $238-622$ & $301-704$ & $430-1,271$ & $244-585$ & $560-1,404$ & 251-609 & $238-622$ \\
\hline
\end{tabular}

Loss of One Amplicon Does Not Strongly Reduce the Analytical Sensitivity of the Triple-Target PCR Assay

It has been shown that the loss of one amplicon in a dual-target approach can result in an underestimation of viral load levels [9]. This motivated us to test whether the failure of one target region in amplification results in a loss of analytical sensitivity of the assay, too. To mimic mutations that lead to detection failure of one target region and to test its effect on dual-target and our triple-target PCR, we withdrew the probe of one of the respective primer/probe sets so that only the amplification of the probe-containing amplicons can be measured. As a control, we run a dualtarget assay containing the identical LTR and pol amplicons. The tests were done using serial dilutions of HIV-1 virus material from tissue culture calibrated against second WHO International Standard for HIV-1 RNA (97/650). Direct comparison of the triple-target with the dual-target approach shows a slightly better analytical sensitivity for the triple-target PCR (table 1, A) which can be attributed to the gag amplicon since the analytical sensitivities of the dual-target and the triple-target assay without the gag amplicon are quite similar (table 1, D). Loss of either the LTR or the pol amplicon strongly reduces the analytical sensitivity of the dual-target assay up to almost twofold, whereas the triple-target PCR is only mildly affected (table 1 , comparing B and C with
A). These experiments clearly demonstrate the higher robustness and safety of the triple-target PCR upon failure of one amplicon compared to a failing amplicon in the dual-target situation and is in good agreement with results published by the PEI, showing a reduced sensitivity in quantitative and qualitative dual-target assays if one amplicon fails $[8,9]$.

Performance of the Triple-Target HIV-1 NAT Assay in the Dayto-Day Routine at Three German Red Cross Blood Donor Service Centres

In January 2014, the first Red Cross blood centre started the routine testing for HIV-1 with the triple-target PCR (VSPK v1.3) followed in February 2014 by the two other blood centres. The three blood centres perform NAT screening of blood products in plasma pools of up to 96 individual samples. The analytical sensitivity $\left(\mathrm{LOD}_{95 \%}\right)$ for HIV-1 RNA for an individual donation present in a pool of 96 donations is $730 \mathrm{IU} / \mathrm{ml}$ (CI 544-1,261; data from GFE Blut) for the autoX 2.0 platform and $800 \mathrm{IU} / \mathrm{ml}$ (CI 556-1,867; data from GFE Blut) for the autoX 1.5 platform. After almost 2 years of routine testing, a total of 3,531,531 donations in 41,793 pools with an average pool size of 85 donations per pool have been tested with the HIV-1 v3 PCR (table 2). Overall, the triple-target PCR performed well, with an average of $0.27 \%$ of invalid pool re- 
Table 2. Results of 2 years of routine blood donation screening with HIV-1 tripletarget PCR

\begin{tabular}{lllll}
\hline & Test site A & Test site B & Test site C & Total \\
\hline Number of donations tested & $1,320,938$ & $1,283,424$ & 927,169 & $3,531,531$ \\
Number of pools tested & 14,005 & 14,029 & 13,759 & 41,793 \\
average size of pools & 94 & 91 & 67 & 85 \\
Number of invalid pools & 27 & 71 & 14 & 112 \\
\% invalid pools & 0.19 & 0.51 & 0.10 & 0.27 \\
Number of initially reactive pools & 24 & 37 & 14 & 75 \\
\% initial reactive pools & 0.17 & 0.26 & 0.10 & 0.18 \\
Number of confirmed positive pools & 13 & 13 & 6 & 32 \\
\% confirmed positive pools & 0.09 & 0.09 & 0.04 & 0.08 \\
Number of NAT-only positive pools & 0 & 0 & 0 & 0 \\
Number of false-positive pools & 11 & 24 & 8 & 43 \\
\% false-positive pools & 0.08 & 0.17 & 0.06 & 0.10 \\
Specificity of HIV-1 triple-target PCR, \% & 99.92 & 99.83 & 99.94 & 99.90 \\
\hline
\end{tabular}

sults and a variation of 0.1 up to $0.51 \%$ among the three blood donation centres (table 2). This value shows a good robustness and reproducibility of the triple-target PCR. The percentage of HIV-1 initial reactive pools was $0.18 \%$ with $0.08 \%$ confirmed HIV- 1 positive pools (table 2). None of these donations were NAT-only-positive. The comprehensive dataset shows a diagnostic specificity of 99.9\% for a total of 9 different lots of VSPK v1.3 being used by the three German blood centres.

\section{Discussion}

Due to the high sequence variability and the fast evolution of new viral variants of HIV-1, test failures were reported from patient diagnostic assays which were mainly based on gag target sequences $[17,18]$ as well as from blood screening assays targeting LTR regions. Thus, the PEI mandated that future blood screening assays must contain at least two independent primer and probe sets. The data published by the PEI revealed that dual-target NAT assays have a reduced sensitivity if one primer/probe set fails. Two dual-target assays were challenged by the PEI with viral isolates from test failure cases (1-6, [9]). One out of the 6 isolates gave a false-negative result with a mono-target assay, whereas both dualtarget assays showed moderately reduced sensitivity (factor $<10$ ) if one amplicon fails to be amplified [9]. It is therefore conceivable that donations contaminated with HIV-1 isolates carrying mismatch mutations in the amplicon regions of the respective NAT assay at a low viral load may be under-quantified or even missed by a dual-target approach.

Having this in mind, we developed a triple-target HIV-1 PCR. Performance data generated with the three single PCRs on synthetic DNA sequences representing consensus sequences and sequences with rare mutations from a set of different subtypes of HIV-1 showed substantial differences in the analytical sensitivities and $\mathrm{Ct}$ values among the different sequences represented by the gBlocks. Amplification of some isolates failed for one amplicon, but at least two amplicons were functional in all of the different sequences (fig. 2). The loss of one amplicon only mildly affected the analytical sensitivity of the triple-target PCR (see table 1), whereas the loss of the same amplicon in the dual-target situation resulted in a rather strong loss of analytical sensitivity (table 1), which can, in a worst case scenario, lead to a false-negative result on a mutated, low virus titre sample. Thus, based on known sequence data including the sequences from the new heavily mutated isolates, the GFE Blut HIV-1 triple-target assay almost fully compensates for the loss of one amplicon, thus adhering even for those isolates and subtypes to the PEI demands.

An intriguing finding was the identification of two independent isolates with a kind of common signature mutations in the LTR. Four identical mutations were observed in the primer binding site region of our withdrawn HIV-1 PCR version 1. Genome sequencing of those isolates revealed their relationship to the clade $B$ subtypes,but with grouping to a separate branch not identified before (fig. 1). The appearance of these new subtype B isolates and its failure to be amplified by our LTR target assay is a good example for the unpredictable risk caused by the failure to detect new (sub) genotypes and/or new recombinant strains that pose a similar risk to every single target assay, no matter whether it concerns the most conserved LTR, gag or pol regions.

Taken together, the advantage of a triple-target NAT assay can be exemplified by its ability to maintain a robust and sensitive target detection even though mutations impairing the primer and probe binding are present in one amplicon region. If two targets remain functional, the triple-target assay can far better compensate for the failure of one amplicon than dual-target NAT tests.

The robustness and performance of our HIV-1 triple-target PCR assay has been confirmed by the data from the routine blood donation screening at three large German Red Cross blood centres (table 2). Altogether, 3.51 million donations have been tested in large pools, showing less than $0.3 \%$ invalid pools and a diagnostic specificity of $99.9 \%$. This confirms the high usability of the HIV-1 v3 PCR for the high-throughput routine blood donation screening.

\section{Disclosure Statement}

The authors declare no conflict of interest relevant to the present manuscript. 


\section{References}

1 Saldanha J, Minor P: A sensitive PCR method for detecting HCV RNA in plasma pools, blood products, and single donations. J Med Virol 1994;43:72-76.

$\checkmark 2$ Roth WK, Weber M, Seifried E: Feasibility and efficacy of routine PCR screening of blood donations for hepatitis $\mathrm{C}$ virus, hepatitis B virus, and HIV-1 in a bloodbank setting. Lancet 1999;353:359-363.

$\checkmark 3$ Roth WK, Busch MP, Schuller A, Ismay S, Cheng A, Seed CR, Jungbauer C, Minsk PM, Sondag-Thull D, Wendel S, Levi JE, Fearon M, Delage G, Xie Y, Jukic I, Turek P, Ullum H, Tefanova V, Tilk M, Reimal R, Castren J, Naukkarinen M, Assal A, Jork C, Hourfar MK, Michel P, Offergeld R, Pichl L, Schmidt M, Schottsted V, Seifried E, Wagner F, Weber-Schehl M, Politis C, Lin CK, Tsoi WC, O'Riordan J, Gottreich A, Shinar E, Yahalom V, Velati C, Satake M, Sanad N, Sisene I, Bon AH, Koppelmann M, Flanagan P, Flesland O, Brojer E, Lętowska M, Nascimento F, Zhiburt E, Chua SS, Teo D, Stezinar SL, Vermeulen M, Reddy R, Park Q, Castro E, Eiras A, Gonzales Fraile I, Torres P, Ekermo B, Niederhauser C, Chen H, Oota S, Brant LJ, Eglin R, Jarvis L, Mohabir L, Brodsky J, Foster G, Jennings C, Notar E, Stramer S, Kessler D, Hillyer C, Kamel H, Katz L, Taylor C, Panzer S, Reesink HW: International survey on NAT testing of blood donations: expanding implementation and yield from 1999 to 2009. Vox Sang 2012;102:82-90.

4 Roth WK, Seifried E: The German experience with NAT. Transfus Med 2002;12:255-258.

5 Hourfar MK, Jork C, Schottstedt V, Weber-Schehl M Brixner V, Busch MP, Geusendam G, Gubbe K, Mahnhardt C, Mayr-Wohlfart U, Pichl L, Roth WK, Schmidt M, Seifried E, Wright DJ; German Red Cross NAT Study Group: Experience of German Red Cross blood donor services with nucleic acids testing: results of screening more than 30 million blood donations for human immunodeficiency virus-1, hepatitis $C$ virus and hepatitis B virus. Transfusion 2008;48:158-166.
Offergeld R, Ritter S, Quabeck L, Hamouda O: HIV-, HCV-, HBV- und Syphilissurveillance unter Blutspendern in Deutschland 2008-2010. Bundesgesundheitsblatt Gesundheitsforschung Gesundheitssch 2012;55: 907-913.

7 Nübling CM, Heiden M, Chudy M, Kress J, Seitz R, Keller-Stanislawsky B, Funk B: Experience of mandatory nucleic acid amplification test (NAT) screening across all blood organizations in Germany: Nat yield versus breakthrough transmissions. Transfusion 2009; 49:1850-1858.

8 Chudy M, Weber-Schehl M, Pichl L, Jork C, Kress J, Heiden M, Funk MB, Nübling CM: Blood screening nucleic acid amplification tests for human immunodeficiency virus Type 1 may require two different amplification targets. Transfusion 2012;52:431-439.

$\checkmark$ Chudy M, Kress J, Halbauer J, Heiden M, Funk MB, Nübling CM: Risk minimization measures for blood screening HIV-1 nucleic acid amplification technique assays in Germany. Transfus Med Hemother 2014;42: 445-451.

10 Müller B, Nübling CM, Kress J, Roth WK, De Zolt S, Pichl L: How safe is safe: new human immunodeficiency virus type 1 variants missed by nucleic acid testing. Transfusion 2013;53:2422-2430.

11 Schmidt M, Korn K, Nuebling CM, Chudy M, Kress J, Horst HA, Geusendam G, Hennig H, Sireis W, Rabenau H, Doerr HW, Berger A, Hourfar MK, Gubbe K, Karl A, Fickenscher H, Tischer K, Babiel R, Seifried E, Guertler L: First transmission of HIV-1 by a cellular blood product after mandatory nucleic acid screening in Germany. Transfusion 2009;49:1836-1844.
12 Paul-Ehrlich-Institut: Bekanntmachung über die $\mathrm{Zu}$ lassung von Arzneimitteln - Abwehr von Arzneimittelrisiken - Vom 15. Juni 2012. Anordnung von Auflagen zu den Zulassungen für zelluläre Blutkomponenten und gefrorene Frischplasmen, hier Anordnung von Maßnahmen zur Risikominimierung beim Einsatz von HIV-1 NAT-Testsystemen. BAnz AT 2012;2.09.2012 B6.

13 Tamura K, Stecher G, Peterson D, Filipski A, Kumar S: MEGA6: Molecular Evolutionary Genetics Analysis version 6.0. Mol Biol Evol 2013;30:2725-2729.

14 Waterhouse AM, Procter JB, Martin DM, Clamp M, Barton GJ: Jalview Version 2 - a multiple sequence alignment editor and analysis workbench. Bioinformatics 2009;25:1189-1191.

15 Zhang M, Schultz AK, Calef C, Kuiken C, Leitner T, Korber B, Morgenstern B, Stanke M: jpHMM at GOBICS: a web server to detect genomic recombinations in HIV-1. Nucleic Acids Res 2006;34:W463-465.

16 Altschul SF, Gish W, Miller W, Myers EW, Lipman DJ: Basic local alignment search tool. J Mol Biol 1990;215: 403-410.

17 Edelmann A, Kalus U, Oltmann A, Stein A, Unbehaun A, Drosten C, Kruger DH, Hofmann J: Improvement of an ultrasensitive human immunodeficiency virus type 1 real-time reverse transcriptase-polymerase chain reaction targeting the long terminal repeat region. Transfusion 2010;50:685-692.

18 Foglieni B, Candotti D, Guarnori I, Raffaele L, Berzuini A, Spreafico M, Orani A, Rossotti R, Rossi D, Allain JP, Prati D: A cluster of human immunodeficiency virus type 1 recombinant form escaping detection by commercial genomic amplification assays. Transfusion 2011;51:719-730. 